\title{
SÍNTESE E ELUCIDAÇÃO ESPECTROSCÓPICA DE NOVOS DERIVADOS 1,2,4-OXADIAZOLÍNICOS, POTENCIAS INIBIDORES DA CICLOOXIGENASE 2
}

\author{
F. N. COUTINHO ${ }^{1}$, E. K. SOUZA ${ }^{1}$, J. A. B. MESQUITA ${ }^{1}$, M. S. de ARAÚJO Neta ${ }^{1}$, \\ A. C. Da SILVA Jr ${ }^{1}$, M. C. Q. De ARAÚJO ${ }^{1}$, L. N. De BARROS ${ }^{1}$, D. R. Da SILVA ${ }^{1}$, \\ T. G. Da SILVA ${ }^{2}$ e A. R. De FARIA*1
}

${ }^{1}$ Universidade Federal de Pernambuco, CCS, Depto. de Ciências Farmacêuticas, Laboratório de Síntese Orgânica Aplicada a Fármacos - LASOF; ${ }^{2}$ Departamento de Antibitóticos-UFPE

E-mail para contato: felipe.nevesc@hotmail.com, *rodolfo.ufpe@gmail.com

RESUMO - Os 1,2,4-oxadiazóis e correlatos, têm demonstrado excelentes atividades biológicas, como antimicrobiana, antitumoral e anti-inflamatória. Recentemente sintetizamos o biciclo pirrolidina[1,2-d]1,2,4-oxadiazolina, contendo a função hidrazona, que apresentou pronunciada atividade antinociceptiva. $O$ objetivo deste trabalho foi a síntese e elucidação de novos derivados hibridos oxadiazolínicos di-arilados com função hidrazona, análogos Coxibes. As iminas formadas, a partir de benzaldeídos e anilinas comerciais, foram submetidas à cicloadição 1,3-dipolar, fornecendo quatro 1,2,4-oxadiazolinas diariladas, com função éster em C3. Suas estruturas foram elucidadas através de ${ }^{1} H$ $R M N,{ }^{13} C R M N$ e IVFT, apresentando um singleto em 6,4ppm ( ${ }^{1} H$ RMN) como sinal diagnóstico da formação do ciclo. Os compostos inéditos tiveram suas propriedades físico-químicas determinadas e a avaliação biológica será posteriormente executada e divulgada.

Palavras-chave: 1,2,4-oxadiazolinas; cicloadição 1,3-dipolar; análogos Coxibe.

ABSTRACT - 1,2,4-oxadiazoles and correlates have shown excellent biological activities, such as antimicrobial, antitumor and anti-inflammatory. We recently have synthesized pyrrolidine[1,2-d]1,2,4-oxadiazoline, containing a hydrazone moiety, which showed pronounced antinociceptive activity. The aim of this work was the synthesis and elucidation of new di-aryl-oxadiazoline/hydrazone hybrids, Coxibes analogs. The starting material, diaryl-imines, were synthesized from benzaldehydes and anilines. 1,3-dipolar cycloaddition between the diaryl-imines and nitrile oxide CEFNO, afforded four diaryl-1,2,4-oxadiazolines, with a C3 ester function. The structures were elucidated by ${ }^{1} \mathrm{H} N M R,{ }^{13} \mathrm{C} N M R$ and IRFT, showing a singlet 6,4ppm ( ${ }^{1} \mathrm{H}$ RMN) as a diagnostic signal of cycle formation. The unprecedented compounds have had their physico-chemical properties determined and the biological evaluation will subsequently be executed and disseminated.

Keywords: 1,2,4-oxadiazolines; 1,3-dipolar cycloaddition; Coxibe analogues. 


\section{INTRODUÇÃO}

Os 1,2,4-oxadiazóis e correlatos, como oxadiazolinas, são compostos amplamente estudados e que em vários trabalhos na literatura demonstraram excelentes resultados no que concerne às suas atividades biológicas, como antimicrobiana, antitumoral e antiinflamatória (RAJAK et al., 2007; ABADI et al, 2003; UNANGST et al, 1992). Recentemente em nosso laboratório, em uma dissertação de mestrado (MENDES, 2010), iniciamos um trabalho que envolve a obtenção de hidrazonas oxadiazolínicas $\underline{\mathbf{2}}$ (figura 1), no qual obtivemos o inédito heterobicíclo, a pirrolidina[1,2-d]1,2,4-oxadiazolina $\underline{\mathbf{1}}$, contendo a função hidrazona, que apresentou pronunciada atividade antinociceptiva, assim como antimicrobiana. Os anti-inflamatórios não esteroidais (AINES) comercias inibem as isoformas da Ciclooxigenase 1 e 2 (COX 1 e 2) de forma não seletiva, com exceção dos Coxibes. A inibição da COX-2 impede a resposta inflamatória, enquanto que a inibição da COX-1 tem causado fenômenos gástricos indesejáveis (UCHÔA, 2009). Desta maneira, pretende-se obter novos derivados híbridos, contendo o núcleo 1,2,4oxadiazolina 4,5-di-arilado $\underline{\mathbf{3}}$ (em vermelho), análogos estruturais de Coxibes, inibidores seletivos da COX-2 e a função hidrazona (em azul), para posterior avaliação das atividades biológicas.

Figura 1: Estruturas contendo o núcleo oxadiazolína. Bicliclos pirrolidina[1.2- $d] 1,2,4-$ oxadiazolina $\underline{\mathbf{1}}$ e hidrazona $\underline{\mathbf{2}}$, híbrido oxadiazolina (vermelho)/hidrazona (azul) $\underline{\mathbf{3}}$.

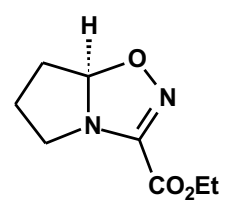

1

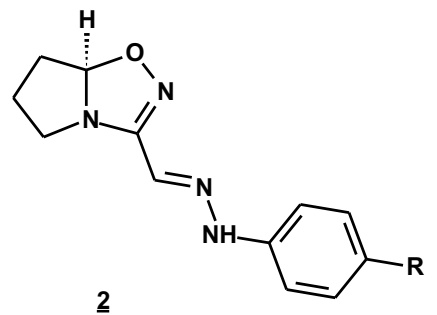

$\underline{2}$

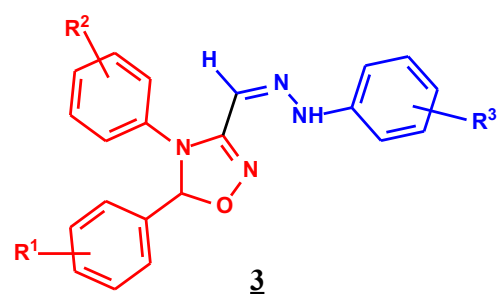

$\underline{3}$

\section{MATERIAIS E MÉTODOS}

As iminas di-ariladas $\underline{\mathbf{5}}$ foram obtidas a partir de reações dos respectivos benzaldeídos substituídos $\underline{4}$ com anilinas substituídas em sonicação, à temperatura ambiente, utilizando $\mathrm{CH}_{2} \mathrm{Cl}_{2}$ como solvente (etapa a). Posterior reações de cicloadição 1,3-dipolar entre as iminas $\underline{\mathbf{5}}$ e o óxido de carboetoxiformonitrila (CEFNO), gerado in situ a partir de seu respectivo precursor (Clorooxiimidoacetato de etila - obtido a partir da glicina esterificada), os cicloadutos 1,2,4-oxadiazolinas di-ariladas $\underline{\mathbf{6}}$ foram obtidos (etapa b). Subsequente redução do éster em $\mathrm{C} 3 \mathrm{com} \mathrm{NaBH}_{4}$ levará à formação do respectivo álcool, que ao ser submetido á oxidação de Swern, levará à formação dos aldeídos oxadiazolínicos $\underline{\mathbf{6}}$ (etapa $\mathrm{c}^{1}$ - redução, etapa $\mathrm{c}^{2}$ - oxidação de Swern). Os aldeídos oxadiazolínicos $\underline{7}$ serão submetidos posteriormente à condensação com diversas semicarbazidas, formando-se assim as oxadiazolinas / hidrazonas híbridas $\underline{\mathbf{3}}$ (etapa d). As semicarbazidas serão obtidas através da reação simples dos respectivos isotiocianatos comerciais com hidrazina. Após purificação em coluna de cromatografia flash, utilizando sílica como fase estacionária, e posterior remoção de resíduos de solvente e água, foram determinadas as características físico-químicas das moléculas e sua elucidação através de análises espectroscópicas de Ressonância Magnética Nuclear ( $\mathrm{RMN}{ }^{1} \mathrm{H}$ e de ${ }^{13} \mathrm{C}$ ) e IVFT.

Esquema 1 - Rota sintética das novas oxadiazolinas di-ariladas/hidrazona $\underline{\mathbf{3}}$ apresentando: benzaldeídos $\underline{\mathbf{4}}$, Iminas di-ariladas $\underline{\mathbf{5}}$, cicloaduto di-arialado oxadiazolina éster $\underline{\mathbf{6}}$, 
cicloaduto di-arilado oxadiazolina aldeído $\underline{7}$.

Etapa a: $\mathrm{CH}_{2} \mathrm{Cl}_{2}, \mathrm{pH}=4-5, \mathrm{MW} /$ Etapa b: $\mathrm{NEt}_{3}, \mathrm{CEFNO}, \mathrm{CHCl}_{3}$, t.a., reação anidra/ Etapa $\mathrm{c}_{1}: \mathrm{NaBH}_{4}, 0^{\circ} \mathrm{C}$, reação anidra $/ \mathrm{c}_{2}$ : DMSO, cloreto de oxalila, $\mathrm{NEt}_{3},-70^{\circ} \mathrm{C}$, reação anidra/Etapa d: EtOH, isocianato, $\mathrm{N}_{2} \mathrm{H}_{4}$.

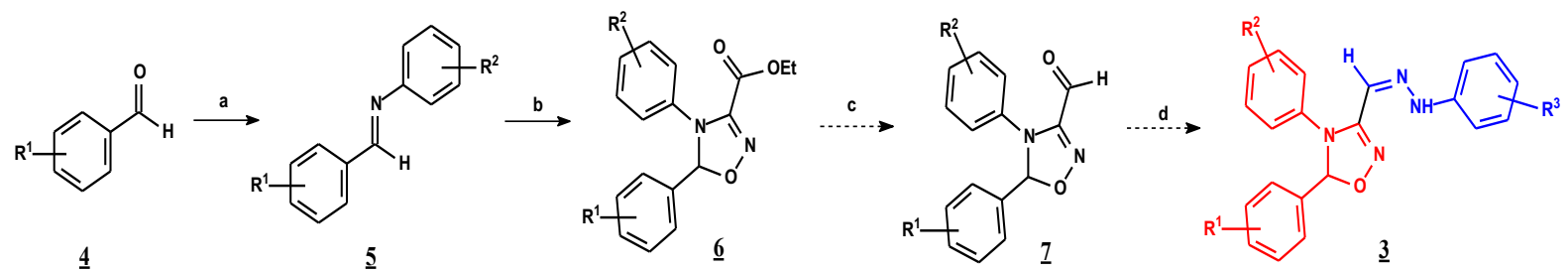

\section{RESULTADOS E DISCUSSÃO}

$\mathrm{Na}$ síntese das iminas di-ariladas foram utilizadas proporções equimolares dos benzaldeídos e anilinas comerciais, utilizando ácido acético como catalisador reacional (McMURRY, 2011). As iminas sintetizadas foram submetidas a reação de cicloadição 1,3 dipolar com o CEFNO, formado in situ (MENDES,2010). Foram obtidos 4 (quatro) novos cicloadutos oxadiazolínicos di-arilados com função éster em C3 apresentados na tabela 1 .

Tabela 1 -Identificação dos substituintes $\mathrm{R}^{1}$ e $\mathrm{R}^{2}$ e características físico-químicas dos compostos oxadiazolínicos di-arilados éster 6a-d sintetizados.

\begin{tabular}{cccccccc}
\hline Código & $\mathrm{R}^{1}$ & $\mathrm{R}^{2}$ & Fórmula molecular & Aspecto & $\begin{array}{c}\text { Rf ActoEt:Hex } \\
(1: 4)\end{array}$ & P.F. ${ }^{\circ} \mathrm{C}$ & Rendimentos \\
\hline$\underline{\mathbf{6 a}}$ & $\mathrm{p}-\mathrm{CH}_{3}$ & $\mathrm{p}-\mathrm{H}$ & $\mathrm{C}_{18} \mathrm{H}_{18} \mathrm{~N}_{2} \mathrm{O}_{3}$ & cristal branco & 0,3 & $110-112$ & $41 \%$ \\
$\underline{\mathbf{6 b}}$ & $\mathrm{p}-\mathrm{NO}_{2}$ & $\mathrm{p}$-isop. & $\mathrm{C}_{20} \mathrm{H}_{21} \mathrm{~N}_{3} \mathrm{O}_{5}$ & óleo avermelhado & 0,36 & óleo t.a. & $29 \%$ \\
$\underline{\mathbf{6 c}}$ & $\mathrm{p}-\mathrm{NO}_{2}$ & $\mathrm{p}-\mathrm{F}$ & $\mathrm{C}_{17} \mathrm{H}_{14} \mathrm{FN}_{3} \mathrm{O}_{5}$ & cristal amarelado & 0,19 & $85-88$ & $28 \%$ \\
$\underline{\mathbf{6 d}}$ & $\mathrm{p}-\mathrm{NO}_{2}$ & $\mathrm{p}-\mathrm{Br}$ & $\mathrm{C}_{17} \mathrm{H}_{14} \mathrm{BrN}_{3} \mathrm{O}_{5}$ & cristal alaranjado & 0,23 & $106-107$ & $23 \%$ \\
\hline
\end{tabular}

A purificação dos cicloadutos $\underline{\text { 6a-d }}$ ocorreu via cromatografia em coluna flash, com sílica como fase estacionária e Acetato de Etila/Hexano como sistema eluente nas proporções apresentadas na tabela 1 . O espectro de $\mathrm{RMN}{ }^{1} \mathrm{H}$ dos compostos $\underline{\mathbf{6} \mathbf{a}}$ e $\underline{\mathbf{6 b}}$ são apresentados nas figuras 2 e 3, tendo um singleto em $6,4 \mathrm{ppm}$, confirmando a formação do ciclo oxadiazolínico, além de outros sinais diagnósticos, que confirmam as estruturas das novas moléculas.

Figura 3: Sinais diagnósticos da oxadiazolina di-aril éster $\underline{\mathbf{6 b}} \mathrm{RMN}{ }^{1} \mathrm{H}\left(\mathrm{CDCl}_{3}\right), 400 \mathrm{MHz}$.
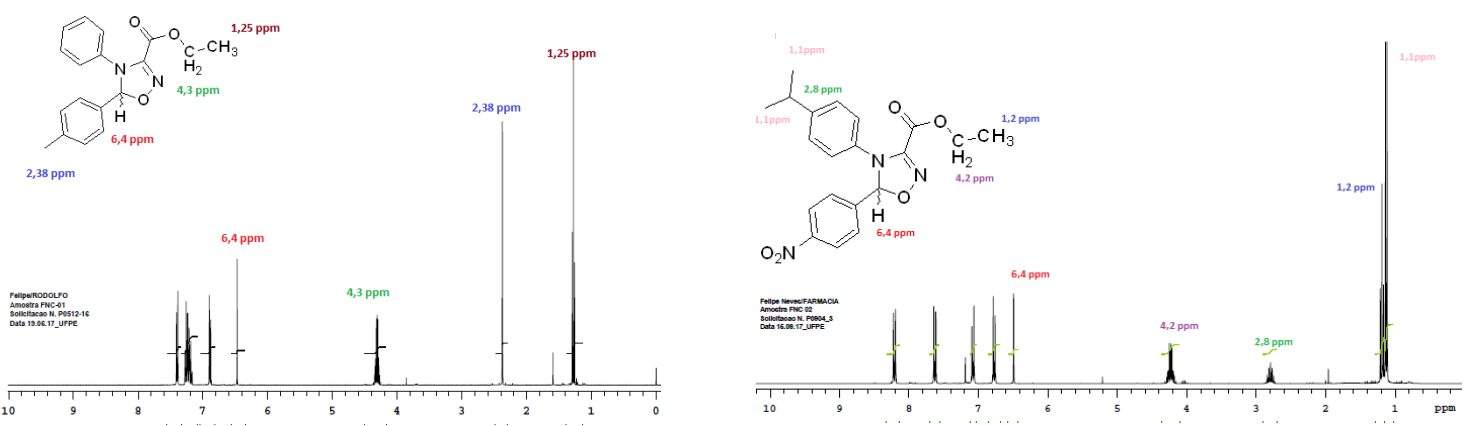


\section{CONCLUSÃO}

INOVAÇÃO TERAPÊUTICA

Os quatro novos compostos 1,2,4-oxadiazolínicos di-arilados 6a-d, com função éster, tiveram suas estruturas confirmadas por $\mathrm{RMN}{ }^{1} \mathrm{H}$ (figuras 2 e 3 ), $\overline{\mathrm{RMN}}{ }^{13} \mathrm{C}$ e IVTF e suas propriedades físico-químicas determinadas. $\mathrm{O}$ estudo da atividade anti-inflamatória in vivo dos análogos Coxibes $\underline{\mathbf{6}}$, na forma de ésteres, está em andamento e a síntese continuará até a obtenção do produto final, o híbrido oxadiazolina/hidrazona.

\section{REFERÊNCIAS}

ABADI, A. H.; EISSA, A. A. H.; HASSAN, G. S. Chem. Pharm. Bull, v. 51, p. 838, 2003.

Charles Christophe Du Barriere Mendes, Dissertação de Mestrado em Ciências Farmacêuticas - UFPE, 2010;

McMURRY, J. Química Orgânica. São Paulo: Editora Cengage Learning, 2011.

RAJAK, H.; KHARYA, M. D; MISHRA, P. Yakugaku Zasshi, v. 127, p. 1757, 2007;

UCHÔA F. T.; DA SILVA T.G.; DE LIMA, M. DO C.; GALDINO, S. L.; PITTA, R.; COSTA T. D. Preclinical pharmacokinetic and pharmacodynamic evaluation of thiazolidinone PG15: an anti-inflammatory candidate. J Pharm Pharmacol. v. 61, p. 339, 2009.

UNANGST, P. C.; SHRUM, G. P.; CONNOR, D. T.; DYER, R. D.; SCHRIER, D. J. J. Med. Chem., v. 35, p. 3691, 1992.

\section{AGRADECIMENTOS}

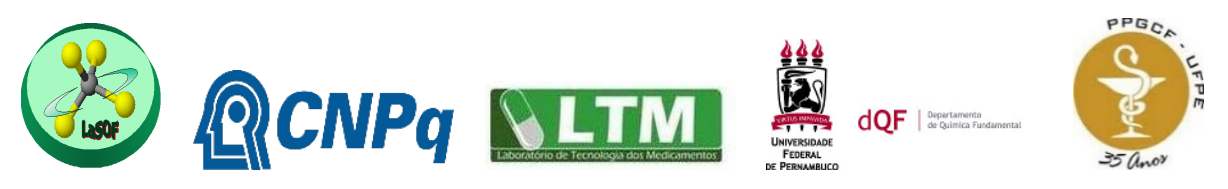

